\title{
Negative Norms in Quantized Strings as Dark Energy Density of the Cosmos*
}

\author{
Mohamed S. El Naschie \\ Dept. of Physics, University of Alexandria, Egypt \\ Email: Chaossf@aol.com \\ Received 5 April 2016; accepted 11 April 2016; published 14 April 2016 \\ Copyright (C) 2016 by author and Scientific Research Publishing Inc. \\ This work is licensed under the Creative Commons Attribution International License (CC BY). \\ http://creativecommons.org/licenses/by/4.0/

c) (i) Open Access

\begin{abstract}
The present short paper is concerned with accurate explanation as well as quantification of the so called missing dark energy of the cosmos. It was always one of the main objectives of any successful general theory of high energy particle physics and quantum cosmology to keep non-physical negative norms, the so called ghosts completely out of that theory. The present work takes the completely contrary view by admitting these supposedly spurious states as part of the physical Hilbert space. It is further shown that rethinking the ghost free condition with the two critical spacetime dimensions $D_{1}=26$ and $D_{2}=25$ together with the corresponding intercept $a_{1}=1$ and $a_{2} \leq 1$ respectively and in addition imposing, as in Gross et al. heterotic superstrings, an overall 496 dimensional exceptional Lie symmetry group, then one will discover that there are two distinct types of energy. The first is positive norm ordinary energy connected to the zero set quantum particles which is very close to the measured ordinary energy density of the cosmos, namely $E(O)=$ $m c^{2} / 22$. The second is negative norm (i.e. ghost) energy connected to the empty set quantum wave and is equal to the conjectured dark energy density of the cosmos $E(D)=m c^{2}(21 / 22)$ presumed to be behind the observed accelerated cosmic expansion. That way we were able to not only explain the physics of dark energy without adding any new concepts or novel additional ingredients but also we were able to compute the dark energy density accurately and in full agreement with measurements and observations.
\end{abstract}

\section{Keywords}

Negative Norms, Quantum Ghosts, Dark Energy, Accelerated Cosmic Expansion, Superstrings, Quantum Field Theory, Cantorian-Fractal Spacetime, Einstein Relativity, Non-Fictional Spurions

\footnotetext{
"Dedicated to the memory of Salah El Naschie, Itidal Rizk, Elsie Thorsen and Trygve Håkon Thorsen who may be called ghosts by some but
} are for me very real. 


\section{Introduction}

One of the most cherished objectives of any successful modern theory in quantum gravity and cosmology is to keep negative norms out of the physical Hilbert space for being meaningless [1]-[5]. However and in view of the shocking discovery of the missing dark energy density of our cosmos as documented beyond reasonable doubt via COBE, WMAP and Type 1a supernova as well as Hubble and Planck projects [6] [7], one may be permitted to contemplate the possibility of having brought on ourselves what may seem superficially to be happening to us as Thomas Mann says in his essay "Freud and the future" [8]. We mean by that the possibility that we have superciliously trimmed and refined our theories to the extent by which we could discover and account for the 4.5 percent ordinary energy only, keeping the 95.5 percent "dark" energy out in the cold under the possibly wrong prejudice that negative norms are spurious terms and mathematical artefacts which have no place in the real world and therefore must be eliminated from the physical Hilbert space using rarefied concepts and mathematics [1]-[5]. The present work motivated by the necessity to explain dark energy turns the tables on the preceding fundamental argument and presents an opposite narrative to the physics of the negative norms which we compute accurately and show it to be what we call dark energy [9]-[19].

\section{Nambu-Goto Strings, Gupta-Bleuler Quantization and Dark Energy}

In the present work we will treat the bosonic section of $D$. Gross et al. heterotic superstrings theory [1]-[5] in the old mould covariant approach of Nambu-Goto bosonic strings and the Gupta-Bleuler quantization of electrodynamics [1]-[5]. Applying the Virasoro algebra to the open strings with almost identical techniques for closed strings, it is well known that due to zero components of the corresponding harmonic oscillation, ghosts are found in theory [1]-[5]. These are given by the negative metric in the usual notation. Thus not only zero norm states but also negative norm states are forced on our mathematics [1]-[5]. However in E-infinity [9]-[15] the zero set models the quantum particle while its surface, i.e. the empty set corresponding to negative norm models the quantum wave [10]. In other words, quantum particles and quantum waves are two different sides of the same coin and are inseparable and moreover, just a mathematical tautology completely equivalent to the zero norm and negative norm [1]-[5] [10]-[14]. Consequently negative norms should not be excluded a priori on account of a rumour, namely being not real in a cosmos where the terms real and not real should be used with a great deal of care and delicacy [1]-[5]. In fact, following the conventional analysis of negative norms, we see that the mathematical scheme itself is trying hard to tell us something but we were not listening, that is until dark energy was conjectured based on real actual measurements and observations [6] [7] [12]. Continuing with this mainstream analysis we can define spurious states leading to an expression which we initially did not want to be part of the physical Hilbert space based on the arguments just being refuted above [1]-[5] [9]-[15]. Said in different words, there is a real possibility that "spurions" as discussed in [16] are real. To make a relatively long story short, following a well known methodology we arrive at a result wrongly conceived as negative, namely that for spacetime dimension $\mathrm{D}=26$ and intercept $a=1.5$, the so called spurious states is part and parcel of our Hilbert space physical structure [1]-[5]. That way we arrive at an acceptable norm state by choosing $D=26$ and can write a ghost free condition prescribing that [1]-[5]

$$
a=1 \text { and } D=26 \text { or } a_{2} \leq 1 \text { and } a \leq 1 D \leq 25 \text {. }
$$

Here we term the $D_{1}=26$ the upper critical dimension while $D_{2}=25$ is the lower critical dimension corresponding to the norm state [1]-[5]

$$
\langle\theta \mid \theta\rangle=\left(a^{2}\right)(2 / 25)(D-1)(26-D) .
$$

Next we will impose our overall 496 dimensional symmetry of E8E8 [1]-[5] and see how we retrieve this way a rather familiar result for the ordinary energy density and consequently the dark energy density $E(D)$ of the cosmos in full agreement with previous analysis and astounding conformity with COBE, WMAP and Type 1a supernova measurements and observations [6] [7] [12].

\section{Internal Dimensions, External Dimensions and Energy Density}

It is well known that the deep meaning of the most important invariance of a topology, namely dimension has transcended the separation between spacetime dimensions and the internal symmetry group dimensions con- 
nected to the same spacetime theory [1]-[5] [9]-[15]. In this sense Einstein's minimum spacetime dimensions $D=$ 4 as well as the 496 dimensions of E8E8 or SO(32) of the two types of heterotic strings as well as the 12 dimensions of SU(3), SU(2), U(1) of the standard model may, at a fundamental level, be treated to a large extent on equal footing [2] [4] [10]. Seen in that way we may look at $D=26$ as an index for positive norm states and consequently link it to quantum particles and ordinary energy. On the other hand $D=496$ may be seen as an index for the infinitely many negative norms and therefore dark energy connected to the empty set, i.e. to the quantum wave [10] [12] [13]. In other words 26/496 must be approximately proportional to the ordinary energy density of our cosmos. To be more precise we need to look at the famous $E=m c^{2}$ of Einstein and to realize that it is the maximal energy density possible, i.e. $E=\gamma_{\max } m c^{2}$ and $\gamma_{\max }=1$ [9]-[15]. We must also realize that $E=m c^{2}$ is based on one messenger particle, namely the photon as well as 4 dimensional spacetime. Now we want to scale $E=\gamma_{\max } m c^{2}$ in accordance with our modern realization of the 12 messenger particles of the standard model (SM) which is all that remains after symmetry breaking from the 496 isometries of the exceptional Lie symmetry group E8E8 representing massless gauge bosons [1]-[5]. To do that our $D=26$ should be reduced to $D=$ $26-4=22$ and our 496 should be reduced to $496-12=484$. Now the density index can be given accurately not by $26 / 496$ but by

$$
\gamma(O)=\frac{D^{(26)}-D^{(4)}}{|E 8 E 8|-S M}=\frac{26-4}{496-12}=\frac{22}{484}=\frac{1}{22} .
$$

Our ordinary energy density is consequently

$$
E(O)=\gamma(O) m c^{2}=m c^{2} / 22
$$

in full agreement with theoretical and experimental results [4]-[15]. The preceding result could be made transfinitely exact [9]-[15] by noting that |E8E8| is not simply 496 but $496-k^{2}$ where $k=2 \phi^{5}=\phi^{3}\left(1-\phi^{3}\right)=0.1833989$ is "tHooft"s hypothetical renormalon and then the $\operatorname{dim} \mathrm{SM}=\operatorname{dim}(\mathrm{SU}(3) \mathrm{SU}(2) \mathrm{U}(1))=12$ may be replaced by the exact topological dimension 4 as well as replacing $D=26$ by the exact value $D=26+k$. Proceeding this way one finds instead of Equation (3) the exact equation [12]-[15]

$$
\gamma(O)=\frac{(26+k)-4}{\left(496-k^{2}\right)-4}=\frac{\phi^{5}}{2}=\frac{1}{22+k}
$$

and

$$
E(O)=m c^{2} /(22+k)
$$

exactly as it should be [9]-[15]. Comparing the previous result with that obtained using the five dimensional volume of the zero set as a quantum particle $\phi^{5}$ and the five dimensional volume of the empty set as a quantum wave $5 \phi^{2}$, i.e. the surface of the 5-D quantum particle, we obtain the very same result [10]-[15]. In other words our final result is that [10]-[15]

$$
E(O)=\left(\phi^{5} / 2\right)\left(m c^{2}\right)=m c^{2} /(22+k) \simeq m c^{2} / 22
$$

which is the quasi potential energy of the quantum particle and this is the energy we can measure directly while [10]-[15]

$$
E(D)=\left(5 \phi^{2} / 2\right)\left(m c^{2}\right)=\left(\frac{21+k}{22+k}\right) m c^{2} \simeq m c^{2}(21 / 22)
$$

is the quasi kinetic propagation energy of the quantum wave enveloping the quantum particle for being its cobordism, i.e. surface. This quasi kinetic energy is actually what we have dubbed the missing dark energy just because we cannot measure it directly due to the quantum wave collapse but only see its effect indirectly via the observed accelerated expansion of the cosmos [6] [7] [12]. In fact we have used more misleading names for the same kind of energy although there are more familiar names as ghost states, spurious states and non-physical states which are then considered not to be admissible parts of the physical Hilbert state. This is for sure a wide spread and misleading understanding which elevated itself wrongly to a conventional wisdom. 


\section{Conclusions}

We were very thorough and successful in clearing our theories from negative norms to the extent that we obscured the origin of dark energy although it is nothing more mysterious than the relatively familiar ghosts, or as said earlier on, the negative norms which we keep saying are not part of reality and should not be part of the physical Hilbert space. Ironically in E-infinity [9]-[15] we worked all the time with empty sets modelling these ghosts but calling them empty sets and called the energy attached to them dark energy and paradoxically referred to it as the energy of the "energy-less empty" quantum wave [9]-[15]. From this muddle now arises at long last a clearer picture. Dark energy is the energy of the quantum wave [9]-[15] [17] and this is actually a tautological expression for the energy of the negative norm states [1]-[5] [16]. In addition locally at very small nano distances, this is the source of the Casimir energy which at the cosmic holographic boundary, is the negative gravity force causing the accelerated cosmic expansion so that the difference between Casimir energy and dark energy is only the difference of boundary conditions and scale [17]. Now even if this is not true, the possibility is truly worth taking seriously and showing why it is not true. However in all events this possibility cannot be dismissed lightly particularly because of its unifying potential and showing again that all our problems in quantum physics and cosmology are connecting to the quantum wave which did not only disturb the great man of physics, Albert Einstein but also one of the main creators of quantum mechanics, E. Schrödinger.

Perhaps when our efforts to develop a quantum wave nondemolition measurement device bear fruitful results, then we will be able to not only deduct dark energy but could utilize it as well as a practically infinite source of clean energy [17]. Maybe we should stress that the present treatment of the subject of dark energy has a particular advantage vis-à-vis all our previous analysis [9]-[15] which is that it does not invoke in the first place any non-mainstream ideas since negative norms and ghosts are fairly familiar concepts [1]-[5] [16] albeit wrongly interpreted in the case of dark energy. In other words we have just show that what we always avoided is what we really need and that quantum waves, empty sets and negative norms are just different words with almost identical meanings [1]-[5] [9]-[15] [17]. Thus we hope that the present paper is written in a language that is familiar to the majority of theoretical physicists.

\section{References}

[1] J. Polchinski: String Theory Vol. I: An Introduction to the Bosonic String. Cambridge University Press, Cambridge, UK, 2000 (See in Particular pp. 16-27).

[2] K. Becker, M. Becker and J.H. Schwarz: String Theory and M-Theory. Cambridge University Press, Cambridge, UK, 2007 (See in Particular pp. 44-51).

[3] M. Kaku: Introduction to Superstrings and M-theory. Springer, New York, USA, 1999. http://dx.doi.org/10.1007/978-1-4612-0543-2

[4] M. Green, J. Schwarz and E. Witten: Superstring Theory. Cambridge University Press, Cambridge, UK 1994 (See in Particular pp. 79-86).

[5] B. Zwiebach: A First Course in String Theory. Cambridge University Press, Cambridge, UK, 2004. http://dx.doi.org/10.1017/CBO9780511841682

[6] L. Amendola and S. Tsujikawa: Dark Energy—Theory and Observations. Cambridge University Press, Cambridge, UK, 2010. http://dx.doi.org/10.1017/CBO9780511750823

[7] P. Ruiz-Lapuente: Dark Energy, Observational and Theoretical Approaches. Cambridge University Press, Cambridge, UK, 2010. http://dx.doi.org/10.1017/CBO9781139193627

[8] Thomas Mann: Freud and The Future (in German: Freud und die Zukunft), Fisher Verlag, Vienna, Austria, 1936.

[9] M.S. El Naschie: Quantum Entanglement: Where Dark Energy and Negative Gravity plus Accelerated Expansion of the Universe Comes From. Journal of Quantum Information Science, 3(2), 2013, pp. 57-77. http://dx.doi.org/10.4236/jqis.2013.32011

[10] M.S. El Naschie: An Exact Mathematical Picture of Quantum Spacetime. Advances in Pure Mathematics, 5, 2015, pp. 560-570. http://dx.doi.org/10.4236/apm.2015.59052

[11] M.S. El Naschie: A Review of E-Infinity and the Mass Spectrum of High Energy Particle Physics. Chaos, Solitons \& Fractals, 19(1), 2004, pp. 209-236. http://dx.doi.org/10.1016/S0960-0779(03)00278-9

[12] M.S. El Naschie: A Unified Newtonian-Relativistic Quantum Resolution of Supposedly Missing Dark Energy of the Cosmos and the Constancy of the Speed of Light. International Journal of Modern Nonlinear Theory \& Application, 2, 2013, pp. 43-54. http://dx.doi.org/10.4236/ijmnta.2013.21005 
[13] L. Marek-Crnjac: On El Naschie’s Fractal-Cantorian Space-Time and Dark Energy-A Tutorial Review. Natural Science, 7(13), 2015, pp. 581-598. http://dx.doi.org/10.4236/ns.2015.713058

[14] M.S. El Naschie: Quantum Dark Energy from the Hyperbolic Transfinite Cantorian Geometry of the Cosmos. Natural Science, 8, 2016, pp. 152-159. http://dx.doi.org/10.4236/ns.2016.83018

[15] M.S. El Naschie: Cosmic Dark Energy Density from Classical Mechanics and Seemingly Redundant Riemannian Finitely Many Tensor Components of Einstein’s General Relativity. World Journal of Mechanics, 4(6), 2014, pp. 153156.

[16] M.D. Schwartz: Quantum Field Theory and the Standard Model. Cambridge University Press, Cambridge, UK, 2014.

[17] M.S. El Naschie: Quantum Fractals and the Casimir-Dark Energy Duality-The Road to a Clean Quantum Energy Nano Reactor. Journal of Modern Physics, 6, 2015, pp. 1321-1333. http://dx.doi.org/10.4236/jmp.2015.69137

[18] M.S. El Naschie: From Witten's 462 Supercharges of 5-D Branes in Eleven Dimensions to the 95.5 Percent Cosmic Dark Energy Density behind the Accelerated Expansion of the Universe. Journal of Quantum Information Science, 6(2), 2016, pp. 57-61.

[19] M.S. El Naschie: On a Fractal Version of Witten’s M-Theory. Journal of Astronomy \& Astrophysics, 6(2), 2016, pp. 135-144. 